\title{
Feedback modeling of non-esterified fatty acids in rats after nicotinic acid infusions
}

\author{
Christine Ahlström • Lambertus A. Peletier • \\ Rasmus Jansson-Löfmark • Johan Gabrielsson
}

Received: 17 August 2010/Accepted: 18 October 2010/Published online: 4 November 2010

(C) The Author(s) 2010. This article is published with open access at Springerlink.com

\begin{abstract}
A feedback model was developed to describe the tolerance and oscillatory rebound seen in non-esterified fatty acid (NEFA) plasma concentrations following intravenous infusions of nicotinic acid (NiAc) to male Sprague-Dawley rats. NiAc was administered as an intravenous infusion over $30 \mathrm{~min}(0,1,5$ or $20 \mu \mathrm{mol} \mathrm{kg} \mathrm{kg}^{-1}$ of body weight) or over $300 \min \left(0,5,10\right.$ or $51 \mu \mathrm{mol} \mathrm{kg}{ }^{-1}$ of body weight), to healthy rats $(n=63)$, and serial arterial blood samples were taken for measurement of NiAc and NEFA plasma concentrations. Data were analyzed using nonlinear mixed effects modeling (NONMEM). The disposition of NiAc was described by a two-compartment model with endogenous turnover rate and two parallel capacity-limited elimination processes. The plasma concentration of NiAc was driving NEFA $(R)$ turnover via an inhibitory drug-mechanism function acting on the formation of NEFA. The NEFA turnover was described by a feedback model with a moderator distributed over a series of transit compartments, where the first compartment $\left(M_{1}\right)$ inhibited the formation of $R$ and the last compartment $\left(M_{N}\right)$ stimulated the loss of $R$. All processes regulating plasma NEFA concentrations were assumed to be captured by the moderator function. The potency, $I C_{50}$, of NiAc was $45 \mathrm{nmol} \mathrm{L}^{-1}$, the fractional turnover rate $k_{\text {out }}$ was $0.41 \mathrm{~L} \mathrm{mmol}^{-1} \min ^{-1}$ and the turnover rate of moderator $k_{t o l}$ was $0.027 \mathrm{~min}^{-1}$. A lower physiological limit of NEFA was modeled as a NiAc-independent release $\left(k_{c a p}\right)$ of NEFA into plasma and was estimated to $0.032 \mathrm{mmol} \mathrm{L}^{-1} \min ^{-1}$. This model can be used to provide
\end{abstract}

C. Ahlström · R. Jansson-Löfmark · J. Gabrielsson

Discovery DMPK and BAC, AstraZeneca R\&D Mölndal, Mölndal, Sweden

C. Ahlström ( $\square)$

Department of Pharmacology, Sahlgrenska Academy at University of Gothenburg,

Gothenburg, Sweden

e-mail: Christine.Ahlstrom@pharm.gu.se

L. A. Peletier

Mathematical Institute, Leiden University, Leiden, The Netherlands 
information about factors that determine the time-course of NEFA response following different modes, rates and routes of administration of NiAc. The proposed model may also serve as a preclinical tool for analyzing and simulating druginduced changes in plasma NEFA concentrations after treatment with NiAc or NiAc analogues.

Keywords Feedback - Pharmacodynamics - Non-esterified fatty acids (NEFA) Rebound · Tolerance $\cdot$ Nicotinic acid $\cdot$ Turnover $\cdot$ Oscillations

\section{Introduction}

Elevated circulating concentrations of non-esterified fatty acids (NEFA) are now recognized as a risk factor in myocardial infarction and occur in patients with myocardial ischemia [1, 2]. Nicotinic acid (NiAc), a lipid lowering agent discovered in the 1950s, inhibits lipolysis in adipose tissue by activating the GPR109A receptor, resulting in a pronounced decrease in plasma concentrations of NEFA [3, 4]. NiAc treatment has been associated with a decrease in cardiovascular events such as myocardial infarction and death [5-7], and to date NiAc is one of few approved drugs that diminishes both low-density lipoprotein (LDL) and triglycerides (TG) and simultaneously elevates high-density lipoprotein (HDL) [7, 8]. Although NiAc has been used for years, its pharmacokinetics and pharmacodynamics are not fully understood. Following NiAc treatment, the change in plasma NEFA concentrations is biphasic, with an initial depression being followed by a rebound elevation to supernormal concentrations when NiAc is eliminated [9]. Furthermore, because of tolerance development there is time dependence in the reduction of plasma NEFA concentrations. For these reasons, it is necessary to clarify the rate and extent of tolerance and rebound development of NEFA with respect to NiAc exposure when optimizing new GPR109A agonists aimed at reducing NEFA.

There have been several approaches to modeling tolerance and rebound including pool/precursor models [10-12], hypothetical counteracting metabolite models [13], and feedback turnover models [14-18]. In this study the latter class of models is applied to the NiAc-induced changes in plasma NEFA concentrations given that these models have proven to be flexible for characterization of the onset, intensity and duration of response [17].

In 2009, our group published a dose-response-time analysis of data from studies where NiAc was administered to rats with different infusion regimens and the changes in plasma NEFA concentrations were measured [18]. The NEFA datasets analyzed were originally designed to qualitatively assess the behavior of plasma NEFA concentrations after different NiAc provocations, rather than for quantitative analysis. As a result, the sampling schedule was intended to detect the maximum and minimum response to $\mathrm{NiAc}$, and to demonstrate the rebound phenomenon. The data collected were more of an exploratory nature than for regression purposes. Furthermore, it was not possible to obtain complete concentration-time profiles of NiAc and NEFA. In the present study, we have refined the experimental design in order to describe the NiAc-NEFA system more thoroughly. 
The primary aim of this study was to develop a feedback model that describes the functional adaptation (tolerance) and oscillatory rebound seen in NEFA plasma concentrations following intravenous infusion of $\mathrm{NiAc}$ at varying rates and duration to Sprague-Dawley rats. Secondary aims were to study the impact of tolerance through graphically visualizing the equilibrium condition for this complex system in relation to the corresponding non-tolerant system, and to describe the nonlinear disposition of NiAc, including its endogenous synthesis.

\section{Materials and methods}

\section{Chemicals}

Nicotinic acid (pyridine-3-carboxylic acid) was obtained from Sigma-Aldrich (St. Louis, MO, USA) and was dissolved in $0.9 \% \mathrm{NaCl}$. All solvents were of analytical grade and the water used in the experiments was obtained from a water purification system (Elgastat Maxima, ELGA, Lane End, UK).

\section{Animals and surgical procedures}

Male Sprague-Dawley rats (Harlan Nederlands B.V.; $n=63$ ) weighing 220-367 g were housed and acclimatized in pairs for at least one week prior to surgery. They had free access to standard rodent chow (R3, Laktamin AB, Stockholm, Sweden) and tap water, and were kept in climate-controlled facilities at a room temperature of $20-22^{\circ} \mathrm{C}$ and relative humidity of $40-60 \%$ under a $12: 12-\mathrm{h}$ light-dark cycle (lights on at 6:00 am). The study was approved by the Ethics Committee for Animal Experiments, Gothenburg, Sweden (EA 100868).

Surgery was performed under isoflurane $\left(\right.$ Forene $^{\circledR}$, Abbott Scandinavia AB, Solna, Sweden) anesthesia and body temperature was maintained at $37^{\circ} \mathrm{C}$ using a thermoregulated heating pad. Catheters were implanted in the left carotid artery for blood sampling and in the right external jugular vein for drug administration, according to the method of Popovic et al. [19]. Prior to cannulation, catheters (Intramedic ${ }^{\circledR}$, PE50, Becton, Dickinson and Company, Franklin Lakes, USA) were filled with sterile sodium-citrate solution (20.6 mM sodium-citrate in sterile saline; Pharmaceutical and Analytical R\&D, AstraZeneca, Mölndal, Sweden) to prevent clotting. After cannulation, the catheters were exteriorized at the nape of the neck and sealed. After surgery, the rats were housed individually and allowed 5-8 days to recover before initiation of the experiments.

\section{Experimental design}

The animals were fasted for $14 \mathrm{~h}$ prior to dosing and throughout the experiment to minimize the fluctuations in NEFA caused by food intake. They had free access to drinking water during the length of fast. On the day of experimentation, they were 
weighed, moved to clean cages, and the venous catheters were connected to infusion pumps (CMA 100, Carnegie Medicin AB, Stockholm, Sweden). Following a $30 \mathrm{~min}$ adaptation period, two consecutive arterial blood samples were collected 15 and 5 min prior to drug administration for determining predose baseline NEFA and NiAc concentrations.

Animals were assigned to 8 groups, each of which received an intravenous constant rate infusion for either 30 or $300 \mathrm{~min}$. Four groups received vehicle $(0.9 \%$ $\mathrm{NaCl}, n=10)$, or $1(n=4), 5(n=8)$ or $20(n=9) \mu \mathrm{mol} \mathrm{kg}{ }^{-1} \mathrm{NiAc}$ over $30 \mathrm{~min}$. The remaining groups received vehicle $(n=8)$, or $5(n=9), 10(n=8)$ or 51 $(n=7) \mu \mathrm{mol} \mathrm{kg}{ }^{-1} \mathrm{NiAc}$ over $300 \mathrm{~min}$. The concentrations of the dosing solutions were adjusted to give infusion volume flow rates in the range of $3.5-22 \mu \mathrm{L} \mathrm{min}{ }^{-1}$, based on body weight. The dosing solutions were prepared within $30 \mathrm{~min}$ of administration by dissolving an appropriate amount of $\mathrm{NiAc}$ in saline solution.

Multiple (11-13 per rat) arterial blood samples were drawn at 2, 5, 10, 20, 28, 32, $35,40,45,50,55,65,85,100 \mathrm{~min}$ for the $30 \mathrm{~min}$ infusion experiments and at 5, 20, $60,120,150,180,240,299,305,315,320,325,340,360,400,420,460$ and $500 \mathrm{~min}$ for the $300 \mathrm{~min}$ infusion experiments for analysis of NiAc and NEFA plasma concentrations. The total blood volume removed did not exceed $1.5 \mathrm{~mL}$, and was replaced with an equal volume of sterile sodium-citrate solution to maintain a constant circulatory volume. The control rats received the same volume of infusion solution (vehicle) as the NiAc groups, and all animals were subjected to similar sampling procedures. The blood samples $(120 \mu \mathrm{L})$ were collected in EDTA coated polyethylene tubes and kept on ice until centrifuged $\left(10,000 \times g, 5 \mathrm{~min}, 4^{\circ} \mathrm{C}\right)$. The plasma was stored at $-20^{\circ} \mathrm{C}$ pending analysis. The start of infusion was taken as time zero $(0 \mathrm{~min})$.

\section{Analytical assays}

Analysis and quantification of NiAc in plasma were done using LC-MS. The high performance liquid chromatography (HPLC) system was an Agilent 1100 Series (Hewlett-Packard GmbH, Walbronn, Germany) coupled to an HTC PAL autosampler (CTC Analytics AG, Zwingen, Germany). Plasma samples (50 $\mu \mathrm{L}$ per sample) were precipitated with cold acetonitrile containing $0.2 \%$ formic acid $\left(150 \mu \mathrm{L}\right.$ per sample). After vortex mixing and centrifugation at $4{ }^{\circ} \mathrm{C}(4,000 \times g$, $20 \mathrm{~min}$ ), an aliquot of $100 \mu \mathrm{L}$ of the supernatant was used for analysis. The mobile phase consisted of (A) $2 \%$ acetonitrile and $0.2 \%$ formic acid in water, and (B) $0.2 \%$ formic acid in acetonitrile. Separation was performed on a $50 \times 2.1 \mathrm{~mm}$ Biobasic AX column with $5 \mu \mathrm{m}$ particles (Thermo Hypersil-Keystone, Runcorn, Cheshire, UK) with a gradient of $95-20 \%$ B over $1 \mathrm{~min}$, held at $20 \% \mathrm{~B}$ for $1.5 \mathrm{~min}$, and returned to initial conditions in one step. The HPLC system was connected to a Sciex API 4000 quadrupole mass spectrometer with a positive electrospray ionization interface (Applied Biosystems, Ontario, Canada) and the mass transition was $124.0>80.2$. Data acquisition and data evaluation were performed using Analyst 1.4.1 (Applied Biosystems). The method showed linearity over a concentration range of $0.001-28 \mu \mathrm{mol} \mathrm{L}^{-1}$. The lower limit of quantification (LLOQ) was $1 \mathrm{nmol} \mathrm{L}^{-1}$ applying a sample volume of $50 \mu \mathrm{L}$ plasma. 
Plasma NEFA was analyzed using an enzymatic colorimetric method (Wako Chemicals GmbH, Neuss, Germany) adapted to a 96-well format [18].

\section{Disposition model of NiAc}

$\mathrm{NiAc}$ is derived from the essential amino acid tryptophan [20]. Endogenous levels of NiAc were detected in a number of predose and control animal samples. The full disposition of NiAc was elucidated by means of exogenous input of the agonist. The aim of modeling NiAc concentration-time data was to obtain individually fitted time-courses that could drive the NEFA concentration-time data. One-compartment models with linear or nonlinear elimination and two-compartment models with linear intercompartmental distribution and linear, nonlinear, parallel linear and nonlinear, or two parallel nonlinear elimination processes from the central compartment, were evaluated. Based on goodness-of-fit (objective function value, OFV) and parameter precision, a two-compartment disposition model with two parallel capacity-limited elimination processes, likely corresponding to glycine conjugation and amidation, and endogenous synthesis (Synt) of NiAc was selected (Fig. 1).

The disposition model of NiAc was mathematically described by:

$$
\begin{gathered}
V_{c} \frac{d C_{p}}{d t}=\operatorname{Inf}+\text { Synt }-\frac{V_{\max _{1}}}{K_{m_{1}}+C_{p}} C_{p}-\frac{V_{\max _{2}}}{K_{m_{2}}+C_{p}} C_{p}-C l_{d} C_{p}+C l_{d} C_{t} \\
V_{t} \frac{d C_{t}}{d t}=C l_{d} C_{p}-C l_{d} C_{t}
\end{gathered}
$$

Fig. 1 Schematic illustration of the feedback model describing the NiAc-induced changes in NEFA. $C_{p}$ and $C_{t}$ denote the NiAc concentrations in plasma and the peripheral compartment. The NiAc disposition parameters are $V_{c}, V_{t}, V_{\max 1}$, $K_{m 1}, V_{\max 2}, K_{m 2}, C l_{d}$, Inf, and Synt (definitions in Table 1). $N E F A$ and $M_{1, \ldots, N}$ denote the response and moderator compartments. The NEFA turnover parameters are $R_{0}, k_{\text {out }}$, $k_{\text {tol }}, k_{\text {cap }}, p, I C_{50}$ and $\gamma$ (definitions in Table 2). $I\left(C_{p}\right)$ is defined in Eq. 2. The number of moderator transit compartments $N$ was 8 . The solid and dashed lines represent fluxes and control processes, respectively
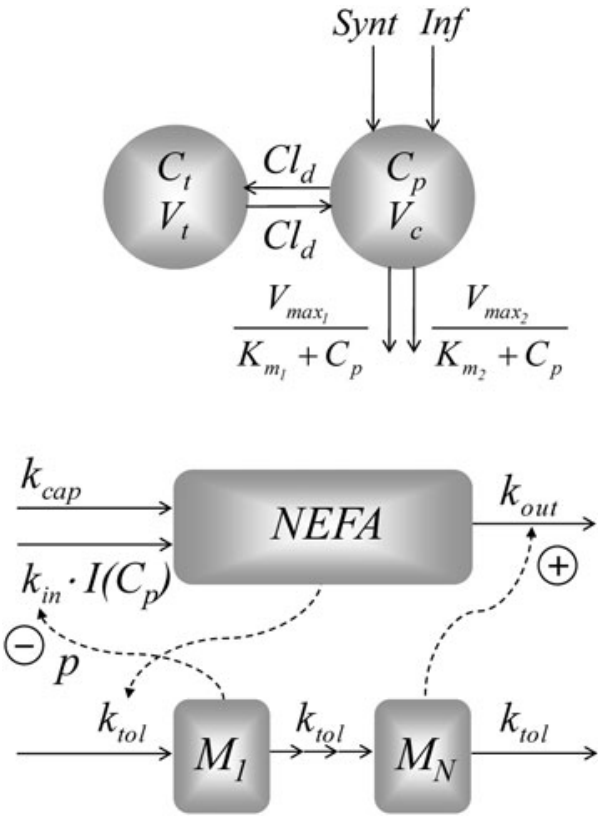
where $C_{p}$ and $C_{t}$ denote the NiAc concentration in the central and peripheral compartment, $V_{c}$ and $V_{t}$ the central and peripheral volume of distribution, Inf the drug infusion rate, Synt the endogenous synthesis rate, $V_{\max 1}$ and $K_{m 1}$ the maximal velocity and Michaelis-Menten constant of the high affinity process, $V_{\max 2}$ and $K_{m 2}$ the maximal velocity and Michaelis-Menten constant of the low affinity process, and $C l_{d}$ the intercompartmental distribution. The endogenous concentration of NiAc was estimated according to Eq. 12 in Appendix A.

Equations $1 \mathrm{a}$ and $1 \mathrm{~b}$ were implemented into the nonlinear mixed effects modeling (NONMEM) program and fitted to the experimental data.

\section{Feedback model of NEFA}

According to the mechanism of action [21-23], NiAc affects plasma NEFA concentrations by inhibition of hydrolysis of TG to NEFA and glycerol in adipocytes, thereby reducing the release of NEFA into plasma. The inhibitory drug mechanism function was described by:

$$
I\left(C_{p}\right)=1-\frac{I_{\max } C_{p}^{\gamma}}{I C_{50}^{\gamma}+C_{p}^{\gamma}}
$$

where $I_{\max }, I C_{50}$ and $\gamma$ are the maximum drug-induced inhibitory effect, plasma concentration at $50 \%$ reduction of maximal effect (potency), and sigmoidicity factor.

The feedback was modeled as a moderator distributed over a series of transit compartments where the moderator in the first compartment, $M_{1}$, inhibited the formation of $R$ (i.e. the build-up of NEFA concentrations). The eighth moderator compartment, $M_{8}$, stimulated the loss of $R$. The dual-action of insulin on NEFA regulation is captured by $M_{1}$ and $M_{8}$, where $M_{1}$ denotes the rapid inhibition of the hydrolysis of TG to NEFA and glycerol and $M_{8}$ the delayed stimulation of re-esterification of NEFA to TG [24-27]. The moderator was affected by $R$ via a first-order process $k_{t o l} R$ and each transduction step used the same transit time $1 / k_{t o l}$. When NiAc inhibits the formation of $R, R$ decreases; consequently the production of moderator $M_{1}$ will decrease. Since the formation of NEFA is inversely proportional to the moderator raised to the power of $p\left(M_{1}^{p}\right)$, the formation of NEFA will thus increase. After a delay, the level of moderator in the final compartment, $M_{8}$, will also drop, resulting in a smaller loss of NEFA. Eventually the concentrations of $R$ and $M_{i}$ (where $i=1, \ldots, 8$ ) will equilibrate.

The hydrolysis of TG to NEFA and glycerol is catalyzed by hormone sensitive lipase (HSL) in adipocytes and by lipoprotein lipase in capillaries. NEFA produced in the capillaries can diffuse into the interstitial space and reach the adipocytes [24]. A fraction of NEFA will remain in the circulation, unaffected by $\mathrm{NiAc}$, representing the lower physiological limit of NEFA in plasma. The hydrolysis of NEFA in the capillaries is incorporated in the model as a zero-order production term, $k_{\text {cap }}$ : 


$$
\frac{d R}{d t}=k_{i n} \frac{1}{M_{1}^{p}} I\left(C_{p}\right)+k_{\text {cap }}-k_{\text {out }} R M_{8}
$$

where $M_{1}$ is the moderator in compartment one, $M_{8}$ the moderator in compartment eight, $k_{i n}$ the turnover rate, $p$ the amplification factor, $I\left(C_{p}\right)$ the inhibitory drug function, $k_{\text {cap }}$ the formation of NEFA in capillaries and $k_{\text {out }}$ the fractional turnover rate. The turnover of the moderators was given by:

$$
\begin{gathered}
\frac{d M_{1}}{d t}=k_{t o l}\left(R-M_{1}\right) \\
\frac{d M_{2}}{d t}=k_{t o l}\left(M_{1}-M_{2}\right) \\
\vdots \\
\frac{d M_{8}}{d t}=k_{t o l}\left(M_{7}-M_{8}\right)
\end{gathered}
$$

The relationship between $R$ and moderator $M_{i}$ at baseline becomes $R_{0}=M_{i, 0}$ and the turnover rate $k_{i n}$ as a function of $R_{0}$ (the baseline NEFA concentration), $k_{\text {out }}$ and $k_{\text {cap }}$ becomes:

$$
k_{\text {in }}=\left(k_{\text {out }} R_{0}^{2}-k_{\text {cap }}\right) R_{0}^{p}
$$

Initial parameter estimates and model selection

The initial estimates of $R_{0}, k_{\text {out }}, k_{\text {cap }}, k_{\text {tol }}$ and $I C_{50}$ were derived graphically. The baseline concentration $R_{0}$ was based on the predose level of NEFA. Following a high dose of NiAc $\left(C_{p} \gg I C_{50}\right)$, Eq. 3 can be approximated by:

$$
\frac{d R}{d t} \approx k_{\text {cap }}-k_{\text {out }} R M_{8}
$$

Provided $k_{\text {cap }}$ is initially much less than $k_{\text {out }} R R_{0}$, Eq. 6 can be simplified to:

$$
\frac{d R}{d t} \approx-k_{\text {out }} R R_{0}
$$

where $M_{8}$ is approximated to $R_{0}$. Thus, the initial downswing of $R$ on a semilogarithmic plot gives a slope of $-k_{\text {out }} R_{0}$. The lower physiological limit of NEFA was reached following the highest dose of NiAc. This allows us to estimate $k_{\text {cap }}$ from:

$$
k_{\text {cap }} \approx k_{\text {out }} R_{s s}^{2}
$$

where $R_{s s}$ denote the steady state response.

We assume that an initial estimate of $k_{t o l}$ may be approximated from the loglinear decline of NEFA post-rebound time-course according to Eq. 30 in Appendix C. The $I C_{50}$ was approximated as the concentration of NiAc resulting in a halfmaximal response following a $30 \mathrm{~min}$ infusion. The sigmoidicity $(\gamma)$ and amplification $(p)$ factors were initially both set equal to unity. 
The number of transit compartments $N$ was obtained by optimizing the objective function value and by visual inspection of the post-rebound oscillations.

The mixed-effects modeling of NiAc exposure and NEFA data was done by means of NONMEM (Version VI level 2.1, Icon Development Solutions, Maryland, USA). The Laplacian estimation method was used throughout the model-building process. Interindividual variability was modeled as exponential models for all disposition parameters of NiAc and NEFA and the random residual variability was modeled as a function of proportional and additive error for the pharmacokinetics and pharmacodynamics. The individual disposition parameters of NiAc were introduced as fixed parameters in the analysis of NEFA data. Model selection was visually explored in diagnostic plots using Census, version 1.1 (Novartis Pharma AG, Basel Switzerland) and based on the OFV and precision of parameter estimates.

\section{Results}

Disposition analysis of NiAc

Observed plasma concentrations of NiAc following infusions of NiAc are shown in Fig. 2. The exposure to NiAc at baseline ranged between 0.001 and $0.07 \mu \mathrm{mol} \mathrm{L}{ }^{-1}$ and approached $20 \mu \mathrm{mol} \mathrm{L}{ }^{-1}$ during the drug infusion regimens. Nonlinear disposition of NiAc was seen in the experimental data following the highest $30 \mathrm{~min}$ infusion dose of $20 \mu \mathrm{mol} \mathrm{kg}{ }^{-1}$. The endogenous NiAc concentration was close to, or below, LLOQ in some animals.

The mixed-effects modeling approach indicated two parallel saturable elimination processes for NiAc. The major clearance pathway up to approximately $0.1 \mu \mathrm{mol} \mathrm{L}^{-1}$ was of high affinity $\left(K_{m 1}=0.00468 \mu \mathrm{mol} \mathrm{L}-1, V_{\max 1}=0.0573 \mu \mathrm{mol} \mathrm{min}{ }^{-1} \mathrm{~kg}^{-1}\right)$. At lower concentrations, the second pathway $\left(K_{m 2}=16.6 \mu \mathrm{mol} \mathrm{L}{ }^{-1}, V_{\max 2}=\right.$ $1.46 \mu \mathrm{mol} \mathrm{min}{ }^{-1} \mathrm{~kg}^{-1}$ ) could be approximated to a first-order process (Fig. 3). The two pathways contributed equally at concentrations around $1 \mu \mathrm{mol} \mathrm{L}{ }^{-1}$ and above that concentration the low affinity pathway became the major elimination process.

The endogenous synthesis of NiAc (Synt) was estimated to $0.0346 \mu \mathrm{mol} \mathrm{min}{ }^{-1} \mathrm{~kg}^{-1}$ and the endogenous NiAc concentration, calculated according to Eq. 12 in Appendix A, was estimated to $6.8 \mathrm{nmol} \mathrm{L}^{-1}$. The shrinkage values were $27 \%$ for $V_{\max 2}, 46 \%$ for $C l_{d}$ and $48 \%$ for Synt. The final NiAc disposition parameter estimates, interindividual variability and their relative standard error (RSE) are shown in Table 1. The two-compartment turnover model (Fig. 1) described the experimental data on an individual bases in a consistent manner (see Fig. 4). The diagnostic plots in Fig. 5 confirmed the consistency of experimental and predicted NiAc concentrations and there were no marked trends in the residual plots.

\section{Feedback model of NEFA}

The observed individual NEFA concentration-time profiles obtained after NiAc infusions are shown in Fig. 6. Baseline NEFA concentrations were consistent in all animals, and were stable throughout infusion of vehicle in the control animals, 


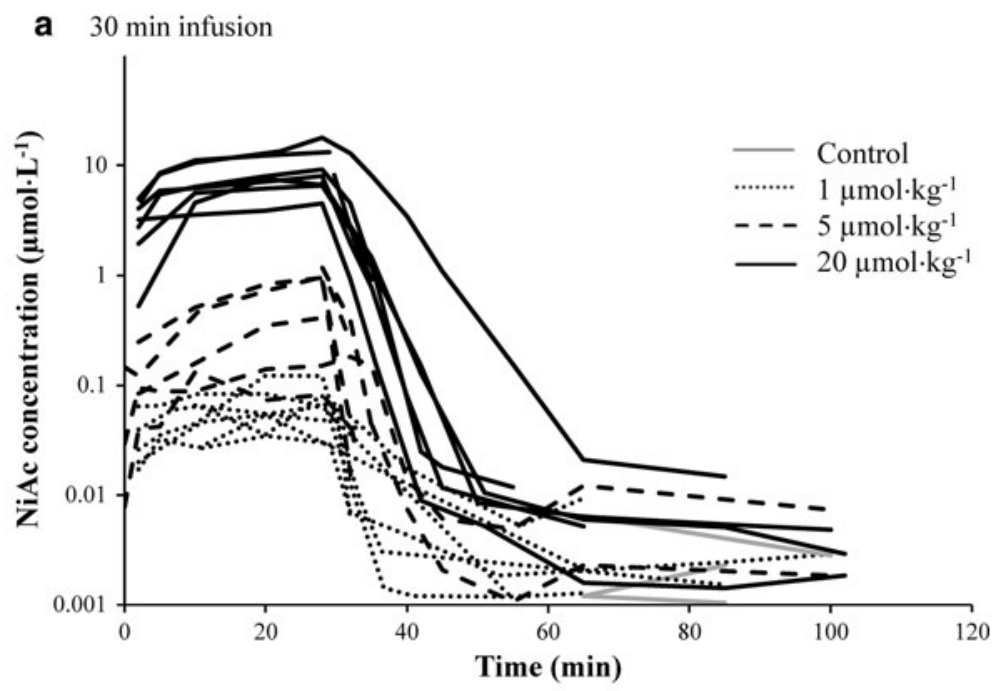

b 300 min infusion

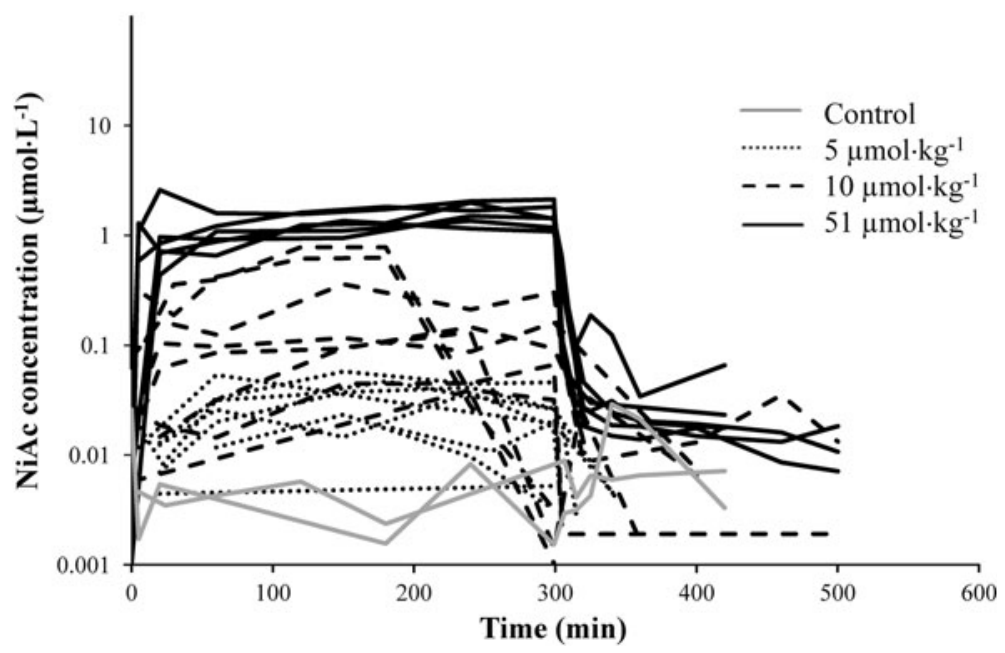

Fig. 2 Observed plasma NiAc concentration-time profiles during and after NiAc infusion. a $30 \mathrm{~min}$ infusion of vehicle (control), or NiAc 1,5 or $20 \mu \mathrm{mol} \mathrm{kg}^{-1}$ of body weight; b $300 \mathrm{~min}$ infusion of vehicle, or NiAc 5,10 or $51 \mu \mathrm{mol} \mathrm{kg}{ }^{-1}$ of body weight. All infusions started at time $t=0 \mathrm{~min}$

ranging between 0.3 and $1.1 \mathrm{mmol} \mathrm{L}^{-1}$ regardless of infusion duration. Administration of NiAc decreased plasma NEFA concentrations to a minimum of $0.055 \mu \mathrm{mol} \mathrm{L}^{-1}$. Following cessation of NiAc infusion, there was a rapid return of NEFA towards baseline, followed by a significant rebound which appeared to be dependent on both the extent and duration of exposure. The amplitude of the rebound was increased following high $\mathrm{NiAc}$ exposure and a post-rebound oscillatory behavior was seen following long (300 min) infusions. A period of 3-4 half-lives of constant 


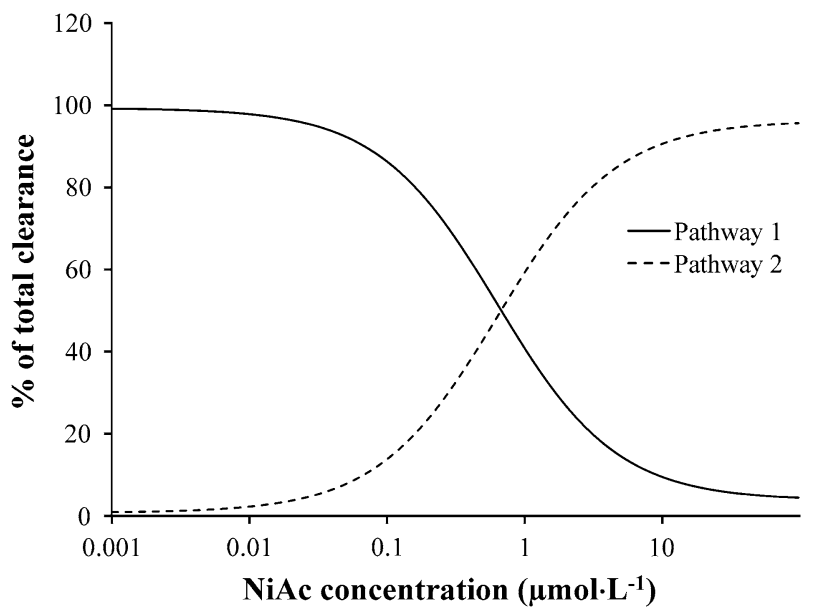

Fig. 3 Saturable elimination processes identified in the disposition of NiAc. The major clearance pathway up to a concentration of approximately $0.1 \mu \mathrm{mol} \mathrm{L}-1$ was high affinity $\left(K_{m 1}=0.00468 \mu \mathrm{mol} \mathrm{L}^{-1}\right.$, $\left.V_{\max 1}=0.0573 \mu \mathrm{mol} \mathrm{min} \mathrm{min}^{-1} \mathrm{~kg}^{-1}\right)$. Below that concentration a second pathway $\left(K_{m 2}=16.6 \mu \mathrm{mol} \mathrm{L}^{-1}\right.$, $V_{\max 2}=1.46 \mu \mathrm{mol} \mathrm{min} \mathrm{mg}^{-1} \mathrm{~kg}^{-1}$ ) could be approximated to a first-order process. The two pathways contributed equally at concentrations around $1 \mu \mathrm{mol} \mathrm{L}^{-1}$ and above that concentration the low affinity pathway became the major elimination process

Table 1 Final NiAc population pharmacokinetic parameter estimates and interindividual variability (IIV), with corresponding relative standard errors (RSE\%)

\begin{tabular}{llrc}
\hline Parameter & Definitions & Estimate (RSE\%) & IIV (RSE\%) \\
\hline$V_{\max 1}\left(\mu \mathrm{mol} \mathrm{min}^{-1} \mathrm{~kg}^{-1}\right)$ & Maximal velocity, pathway 1 & $0.0573(1.57)$ & - \\
$\left.K_{m 1}(\mu \mathrm{mol} \mathrm{L})^{-1}\right)$ & Michaelis-Menten constant, pathway 1 & $0.00468(1.97)$ & - \\
$V_{\max 2}\left(\mu \mathrm{mol} \mathrm{min}^{-1} \mathrm{~kg}^{-1}\right)$ & Maximal velocity, pathway 2 & $1.46(4.20)$ & $98.2(24.8)$ \\
$\left.K_{m 2}(\mu \mathrm{mol} \mathrm{L})^{-1}\right)$ & Michaelis-Menten constant, pathway 2 & $16.6(6.20)$ & - \\
$V_{c}\left(\mathrm{~L} \mathrm{~kg}^{-1}\right)$ & Volume, central compartment & $0.345(0.559)$ & - \\
$C l_{d}\left(\mathrm{~L} \mathrm{~min}^{-1} \mathrm{~kg}^{-1}\right)$ & Intercompartmental clearance & $0.0203(3.08)$ & $54.5(88.6)$ \\
$V_{t}\left(\mathrm{~L} \mathrm{~kg}^{-1}\right)$ & Volume, peripheral compartment & $3.54(7.66)$ & - \\
$S y n t\left(\mu \mathrm{mol} \mathrm{min}^{-1} \mathrm{~kg}^{-1}\right)$ & Endogenous synthesis rate & $0.0346(4.10)$ & $15.8(62.5)$ \\
$\sigma_{1}(\%)$ & Residual proportional error & $34.4(18.6)$ & - \\
$\sigma_{2}(\%)$ & Residual additive error & $0.800(5.80)$ & - \\
\hline
\end{tabular}

NiAc exposure is needed to develop full tolerance. However, due to the 12 min halflife of $k_{t o l}$ (Eq. 30) and the limited resolution of the experimental data during the 30 min infusion, clear adaptation could not be seen.

Figure 7 shows representative individual and population predictions superimposed on experimental data after different doses and infusion times. Modelpredicted and experimental data were consistent. The present design showed adaptation, rebound and post-rebound oscillatory behavior in NEFA concentrations.

The final population parameter estimates and interindividual variability are shown in Table 2 . The half-life of the primary effect $\left(t_{1 / 2, \text { kout }}\right)$ was estimated to 

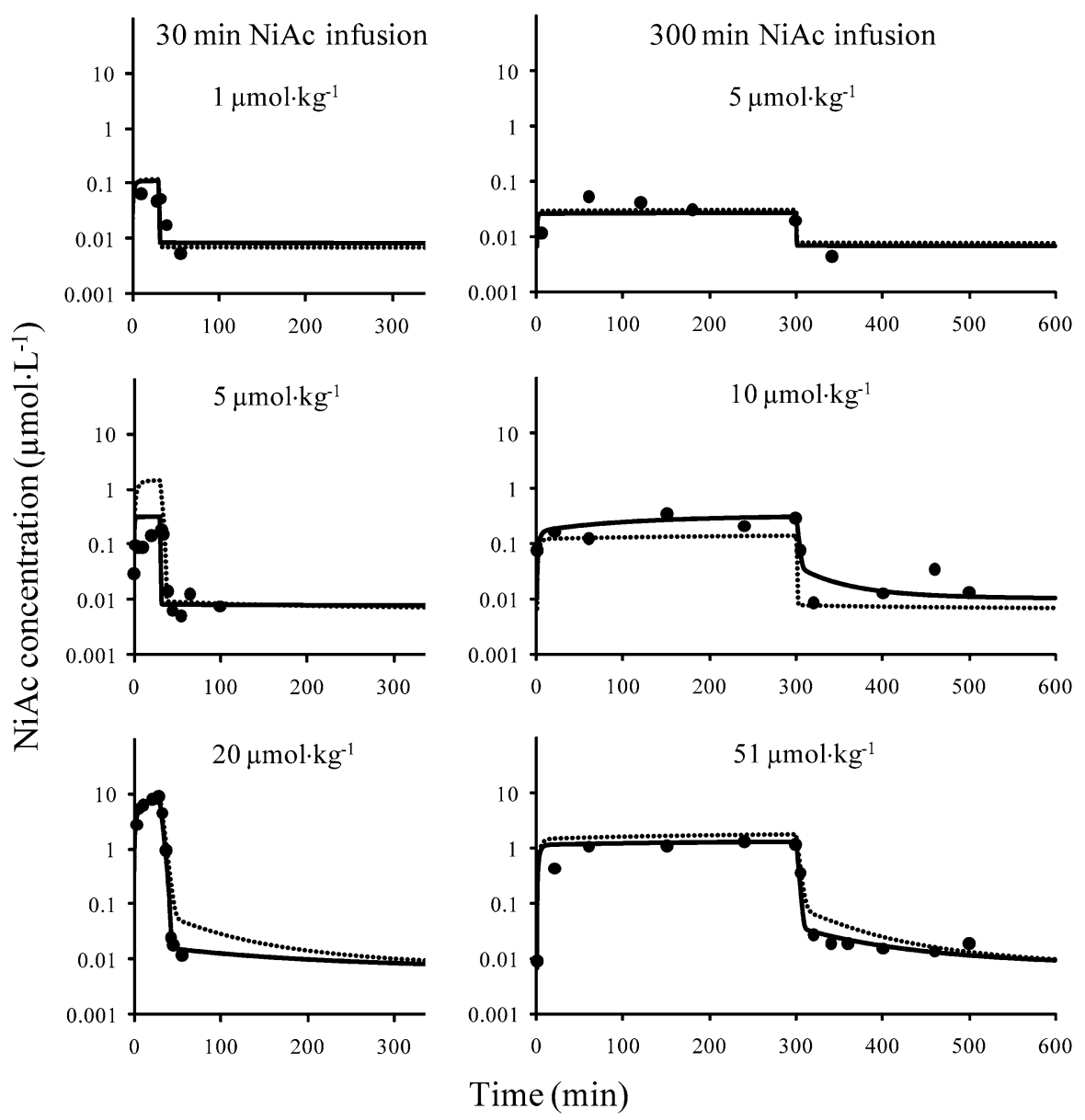

Fig. 4 Representative model fits of NiAc plasma concentration-time data at different durations (30 min (left) and $300 \mathrm{~min}$ (right)) and rates of NiAc infusion. Solid and dashed lines represents individual and population fits, respectively. Infusion started at time $t=0 \mathrm{~min}$. Plots of all individual regressions are available from the author upon request

$1.7 \mathrm{~min}$ and the half-life of tolerance development $\left(t_{1 / 2, k t o l}\right)$ was estimated to $12 \mathrm{~min}$. The shrinkage values were $27 \%$ for $R_{0}, 13 \%$ for $k_{\text {out }}$ and $4 \%$ for $I C_{50}$. The diagnostic plots in Fig. 8 confirmed the consistency of experimental and predicted NEFA concentrations. There were no marked trends in the residual plots. Random residual variability was originally modeled as a function of proportional and additive error. However, as the estimated additive residual error was negligible, only proportional error was used.

The optimal number of moderator transit compartments $N$ was estimated to be 8 .

The equilibrium NiAc concentration-NEFA response relationships of both a tolerant and non-tolerant system (Fig. 9) were simulated using the final parameter estimates (Table 2, Eqs. 13-20 in Appendix B). In the tolerant system the 

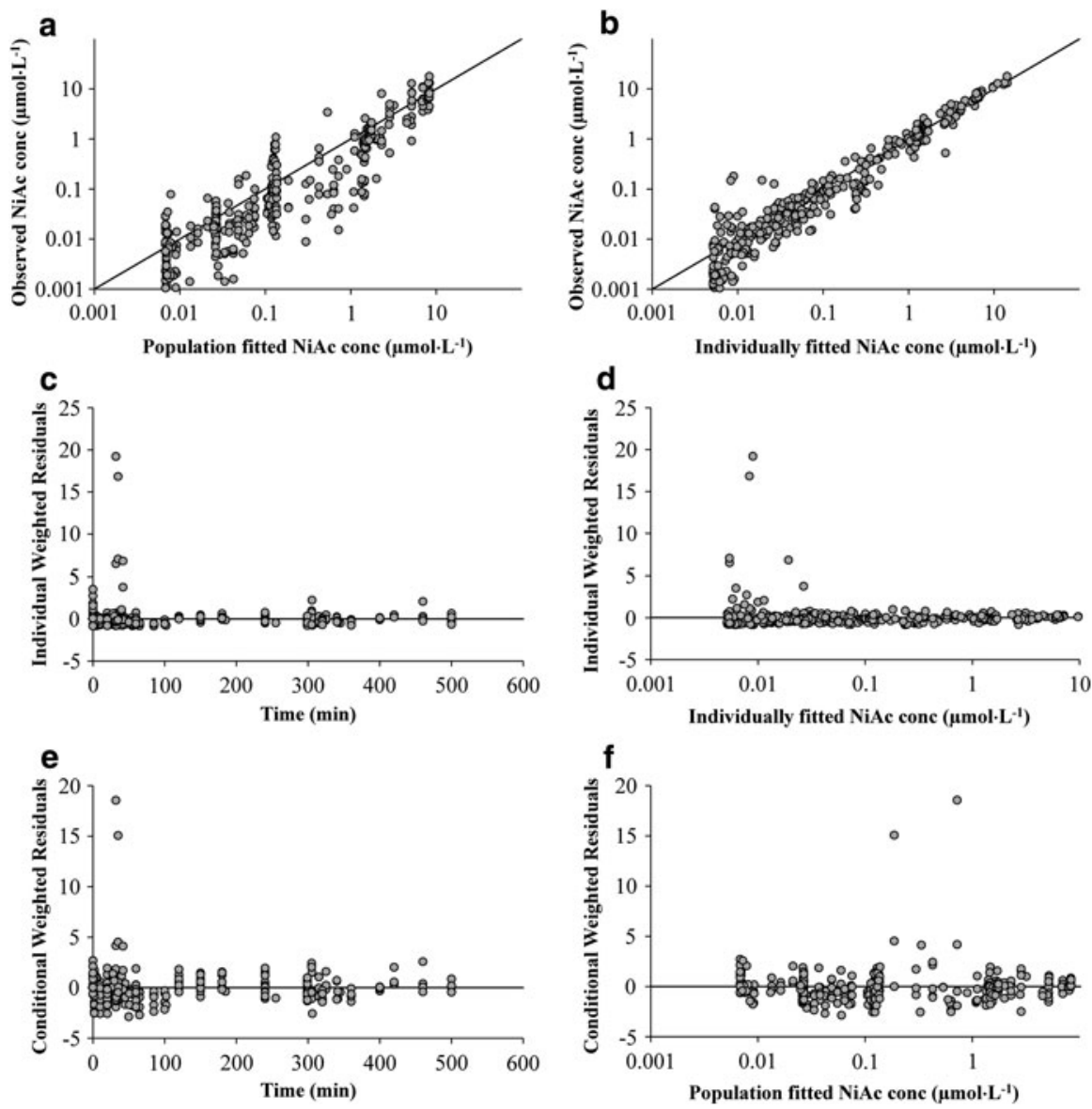

Fig. 5 Goodness-of-fit plots for NiAc. Measured concentrations were plotted against population fitted concentrations (a) and individually fitted concentrations (b) on a logarithmic scale. Individually weighted residuals were plotted against time (c), and against individually fitted NiAc concentrations on a semilogarithmic scale (d). Conditional weighted residuals were plotted against time (e), and against population fitted concentrations (f)

relationship was shallower (shifted upwards) and shifted to the right compared to a non-tolerant system.

\section{Discussion}

NiAc treatment has been reported to decrease cardiovascular events such as myocardial infarction and death [5-7] by altering the lipoprotein profile in the circulation. When NiAc binds to the G-protein-coupled receptor GPR109A in adipocytes, the hydrolysis of TG to NEFA and glycerol is inhibited. The decreased plasma NEFA concentrations are followed by a decrease in plasma TG, very-lowdensity lipoproteins (VLDL) and LDLs, and an increase in plasma HDLs [7, 8]. 


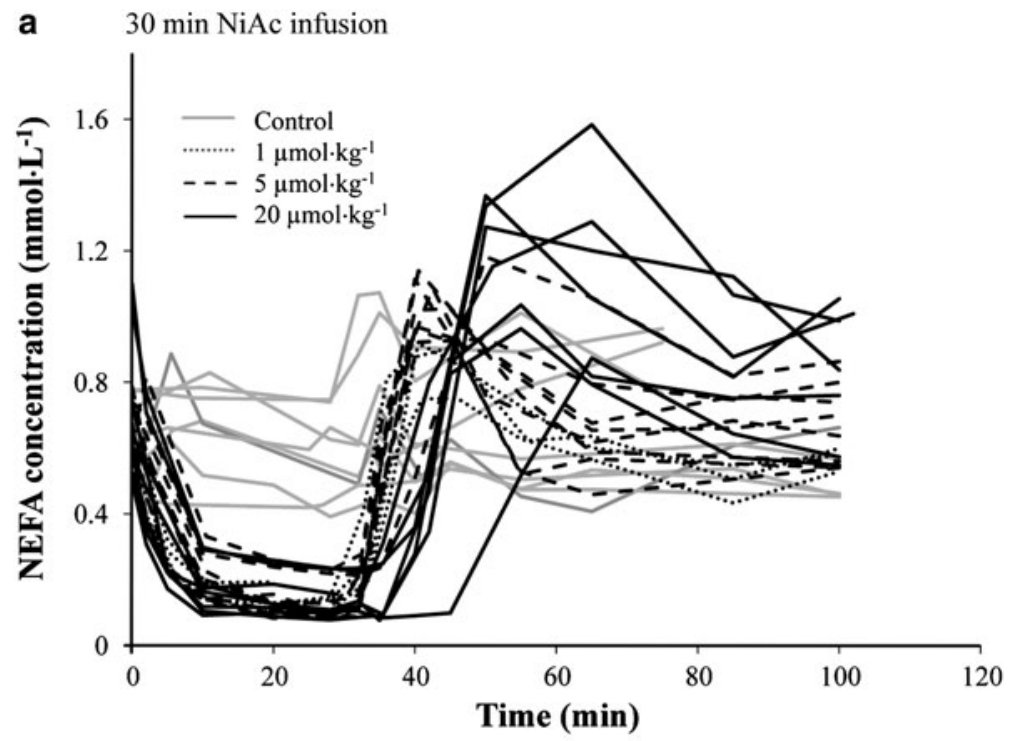

b $\quad 300 \mathrm{~min} \mathrm{NiAc}$ infusion

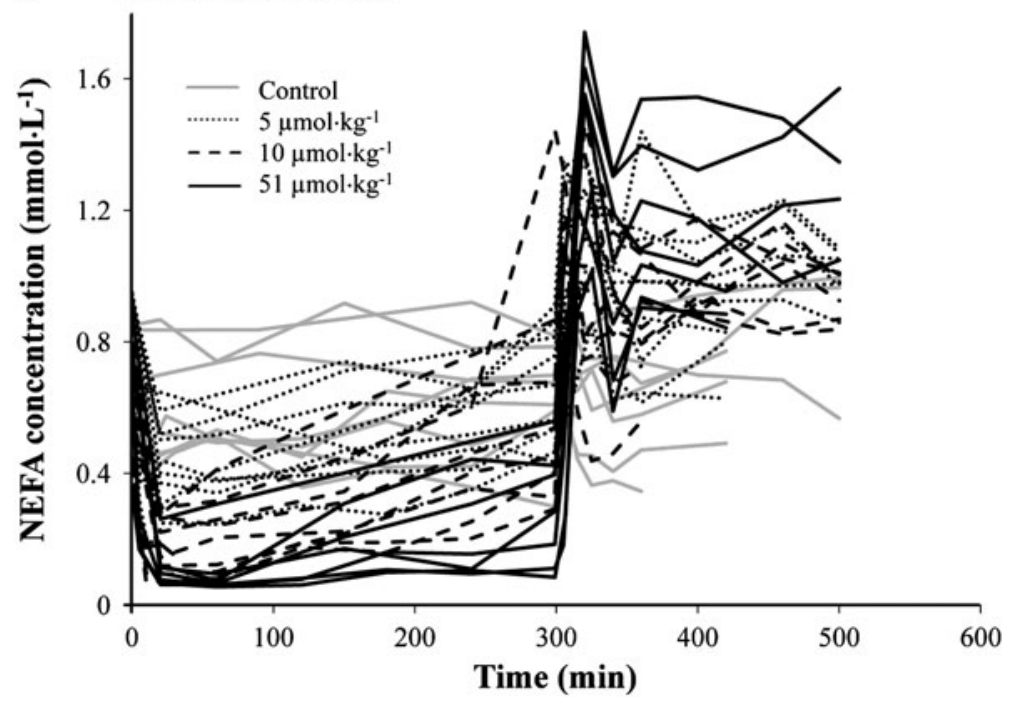

Fig. 6 Observed plasma NEFA concentration-time profiles during and after a a 30 min infusion of vehicle or NiAc $\left(1,5\right.$ or $20 \mu \mathrm{mol} \mathrm{kg}{ }^{-1}$ of body weight), and $\mathbf{b}$ a $300 \mathrm{~min}$ infusion of vehicle or NiAc (5, 10 or $51 \mu \mathrm{mol} \mathrm{kg}{ }^{-1}$ of body weight). The NEFA concentrations in each one of the control animals were stable but a large variability could be seen between the animals. All infusions started at time $t=0 \mathrm{~min}$

However, use of NiAc is limited because it is associated with a substantial rebound of plasma NEFA concentrations above baseline levels, increasing the risk of myocardial ischemia $[1,2]$. For this reason, there is a need to better understand the mechanisms behind the complex regulation of plasma NEFA metabolism and its feedback mechanisms (e.g. insulin). This is particularly so in a drug discovery 

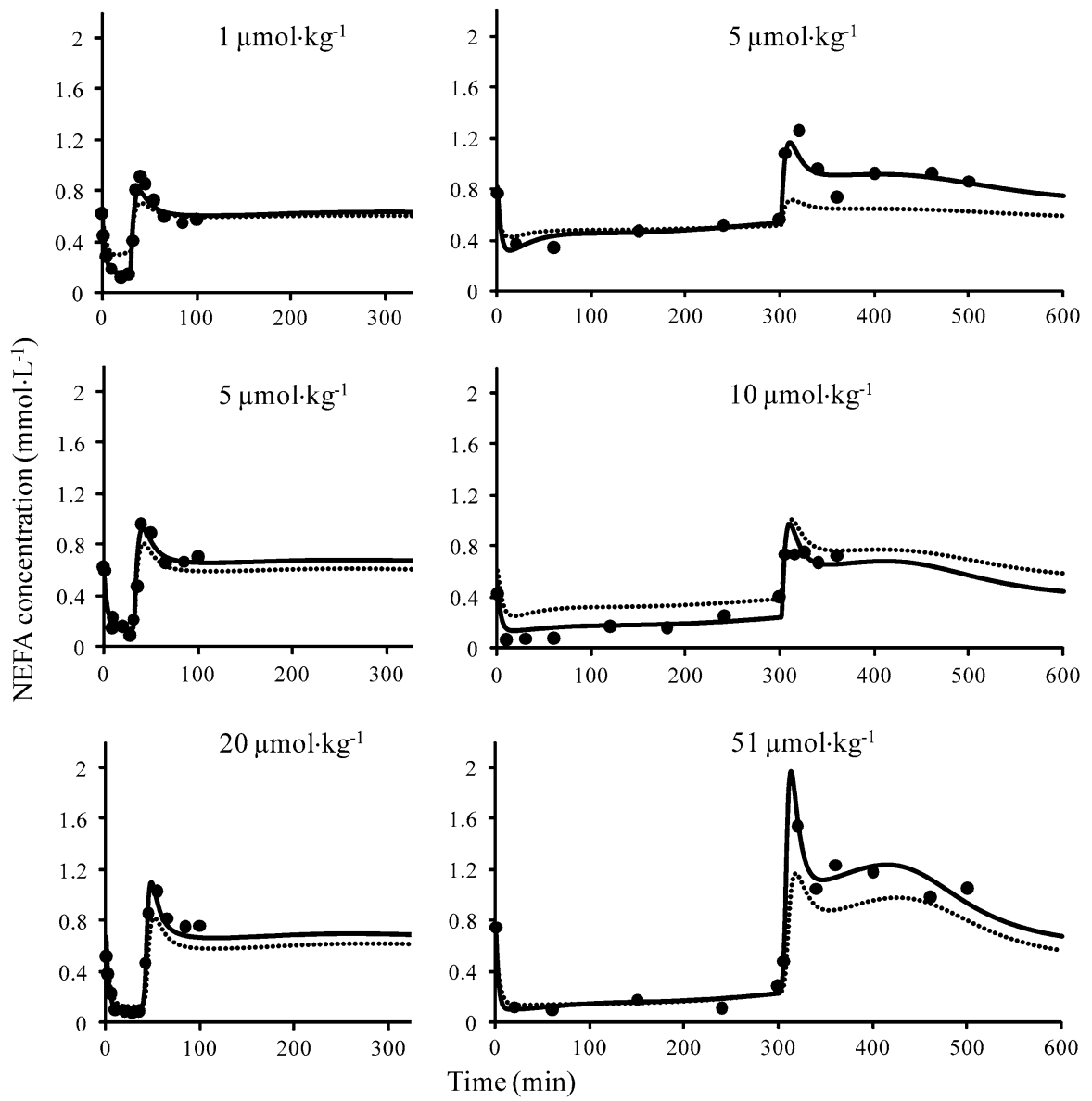

Fig. 7 Representative model fits of NEFA plasma concentration-time data after different durations (30 $\mathrm{min}$ (left) and $300 \mathrm{~min}$ (right)) and rates of NiAc. Solid and dashed lines represent individual and population fits, respectively. Infusion started at time $t=0 \mathrm{~min}$. Plots of all individual regressions are available from the author upon request

setting, when rapid screening decisions need to be based on robust and predictive in vivo models. For example, potential functional adaptation and rebound need to be studied during evaluation of NiAc analogues, as these may confound the assessment of target engagement of new compounds [28]. Thorough quantitative analysis of the time-course of the plasma NEFA response to NiAc in normal or diseased animals provides one source of important information about this system.

Since normal animals provide the primary screening model, it was necessary to first investigate the disposition of $\mathrm{NiAc}$ at pharmacological exposure levels. Exposure to NiAc ranged from 0.001 to about $20 \mu \mathrm{mol} \mathrm{L}^{-1}$ in the infusion groups (Fig. 2), and disposition was clearly nonlinear following the highest $30 \mathrm{~min}$ infusion $\left(20 \mu \mathrm{mol} \mathrm{kg}{ }^{-1}\right)$. We successfully fitted a two-compartment model with two parallel capacity-limited elimination processes, corresponding to glycine conjugation and 
Table 2 Population pharmacodynamic (NEFA response) parameter estimates and interindividual variability (IIV) with corresponding relative standard errors (RSE\%)

\begin{tabular}{llcc}
\hline Parameter & Definitions & Estimate (RSE\%) & IIV (RSE\%) \\
\hline$R_{0}\left(\mathrm{mmol} \mathrm{L}^{-1}\right)$ & Baseline NEFA concentration & $0.606(3.51)$ & $29.0(43.1)$ \\
$k_{\text {out }}\left(\mathrm{L} \mathrm{mmol}^{-1} \mathrm{~min}^{-1}\right)$ & Fractional turnover rate & $0.411(9.22)$ & $48.2(35.6)$ \\
$k_{\text {tol }}\left(\mathrm{min}^{-1}\right)$ & Turnover rate of moderator & $0.0267(3.40)$ & - \\
$\gamma$ & Sigmoidicity factor & $1.48(3.79)$ & - \\
$I C_{50}\left(\mu \mathrm{mol} \mathrm{L}^{-1}\right)$ & Potency & $0.0446(7.20)$ & $101(46.1)$ \\
$p$ & Amplification factor & $1.21(6.64)$ & - \\
$k_{\text {cap }}\left(\mathrm{mmol} \mathrm{L}^{-1} \mathrm{~min}^{-1}\right)$ & Formation of NEFA in capillaries & $0.0318(6.51)$ & - \\
$k_{\text {in }}\left(\mathrm{mmol} \mathrm{L}^{-2} \mathrm{~min}^{-1}\right)$ & Turnover rate of NEFA & 0.0650 & - \\
$t_{1 / 2, \text { kout }}(\mathrm{min})$ & Half-life of primary response & 1.69 & - \\
$t_{1 / 2, \text { ktol }}(\mathrm{min})$ & Half-life of tolerance & 11.7 & - \\
$I_{\text {max }}$ & Efficacy & 1 & - \\
$\sigma(\%)$ & Residual proportional error & $22.1(8.51)$ & - \\
\hline
\end{tabular}

amidation, and endogenous synthesis to the NiAc data. Although a model with dual saturable elimination pathways was proposed by Iwaki et al. [29], their model failed to converge. The glycine conjugation forming nicotinuric acid (NiUAc) was shown by Iwaki et al. to be capacity-limited, and there was also weak evidence that the amidation pathway was capacity-limited. Because a two-compartment model with a single capacity-limited elimination pathway failed in our study, we used a model with two parallel capacity-limited pathways. The estimates of $V_{\max }$ and $K_{m}$ were $0.0573 \mu \mathrm{mol} \mathrm{min}-1 \mathrm{~kg}^{-1}$ and $0.00468 \mu \mathrm{mol} \mathrm{L}^{-1}$ for the high affinity process, and $1.46 \mu \mathrm{mol} \mathrm{min}^{-1} \mathrm{~kg}^{-1}$ and $16.6 \mu \mathrm{mol} \mathrm{L}{ }^{-1}$ for the low affinity process. The high affinity/low capacity process was the major clearance pathway up to approximately $0.1 \mu \mathrm{mol} \mathrm{L}{ }^{-1}$ and the two processes contributed equally at concentrations around $1 \mu \mathrm{mol} \mathrm{L}^{-1}$ (Fig. 3). Iwaki et al. [29] obtained a $V_{\max }$ and $K_{m}$ of $1.9 \mu \mathrm{mol} \mathrm{\textrm {min } ^ { - 1 } \mathrm { kg } ^ { - 1 } \text { and } 3 9 \mu \mathrm { mol } \mathrm { L }}{ }^{-1}$, respectively. The discrepancy in $K_{m}$ may be due to the different concentration ranges (Iwaki et al. approximately $0.8-800 \mu \mathrm{mol} \mathrm{L}{ }^{-1}$, present study approximately $0.001-20 \mu \mathrm{mol} \mathrm{L}{ }^{-1}$ ), with the estimated $K_{m 2}$ value in our study being close to the highest measured NiAc concentration. In addition, Iwaki et al. did not include an endogenous synthesis parameter, nor did they use a mixed-effects modeling approach. The use of different rat strains (Wistar by Iwaki et al., Sprague-Dawley in the present study) and nutritional state are also potential sources of variation between these two studies. Finally, the LLOQ was $0.001 \mu \mathrm{mol} \mathrm{L}^{-1}$ in our study, and $0.8 \mu \mathrm{mol} \mathrm{L}^{-1}$ in the Iwaki et al. study.

The disposition model described the observed individual NiAc data accurately and the model parameters were estimated with good precision. The interindividual variability in some parameters was large. However, the experimental NiAc concentration-time profiles in Fig. 2 showed large interindividual variability in concentration both at baseline and at steady state. The individually predicted NiAc 

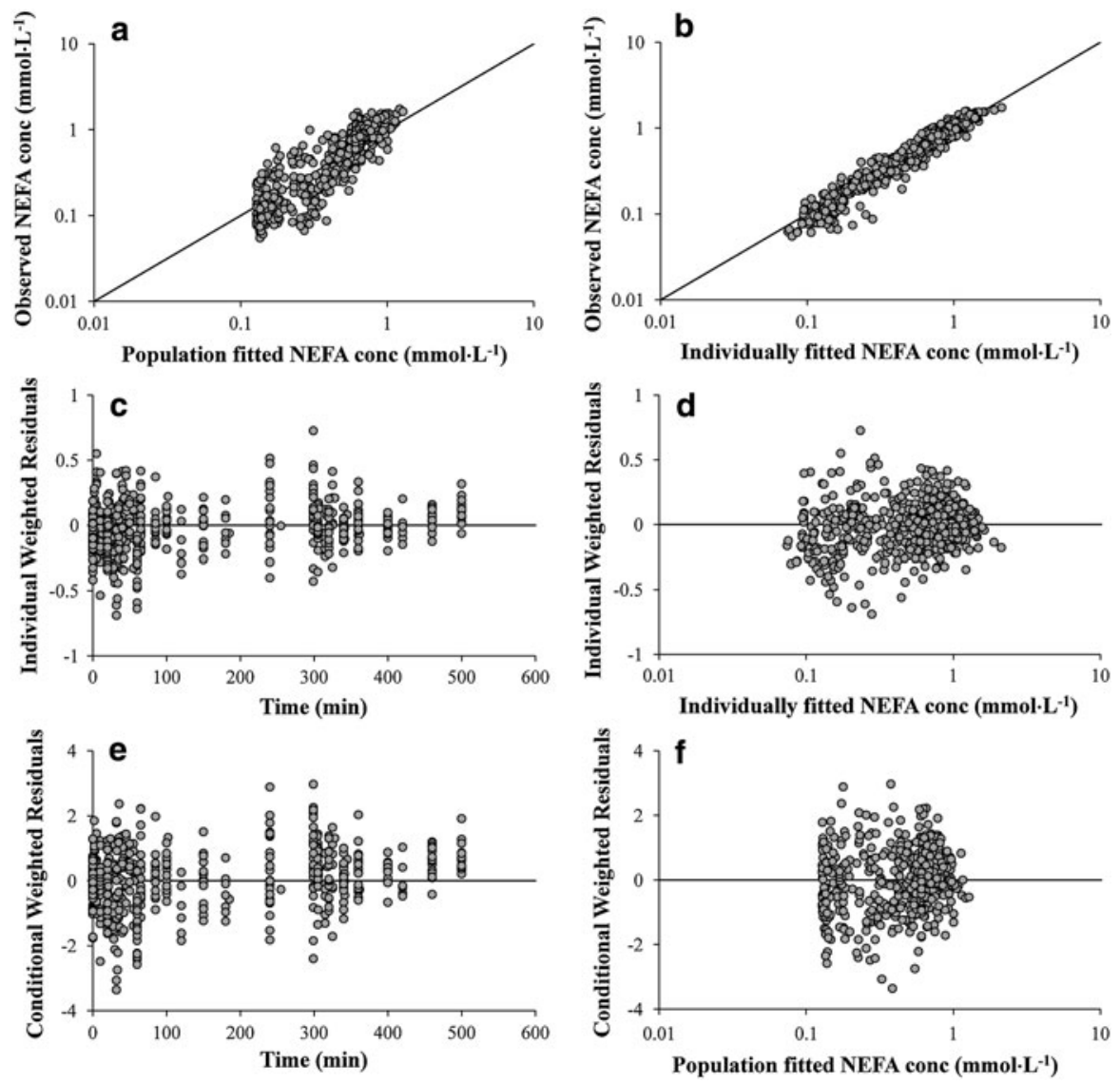

Fig. 8 Goodness-of-fit plots for NEFA. Measured concentrations were plotted against population fitted concentrations (a) and individually fitted concentrations (b) on a logarithmic scale. Individual weighted residuals were plotted against time (c), and against individually fitted NEFA concentrations on a semilogarithmic scale (d). Conditional weighted residuals were plotted against time (e), and against population fitted concentrations (f)

concentration time-courses were consistent with the experimental data (Figs. 4, 5) and therefore fulfilled its primary objective as a smoothing function.

We estimated endogenous synthesis of NiAc (Synt) to be $0.0346 \mu \mathrm{mol} \mathrm{min}{ }^{-1} \mathrm{~kg}^{-1}$ (population range $0.0297-0.0439 \mu \mathrm{mol} \mathrm{min} \mathrm{mg}^{-1} \mathrm{~kg}^{-1}$ ), resulting in a baseline concentration of $6.8 \mathrm{nmol} \mathrm{L}^{-1}$ (population range $4.8-15 \mathrm{nmol} \mathrm{L}^{-1}$ ). However, the endogenous concentration of NiAc was not measurable in all predose and control group samples which may have biased the results.

Infusion of NiAc effectively decreased the plasma NEFA concentration in rats to around $10 \%$ of the predose baseline level (Fig. 6). Increasing the infusion rate from 0.17 to $0.67 \mu \mathrm{mol} \mathrm{min}{ }^{-1} \mathrm{~kg}^{-1}$ did not further decrease NEFA concentrations. This suggests a lower limit of NEFA concentrations at $0.055 \mu \mathrm{mol} \mathrm{L}^{-1}$. After stopping NiAc infusion, plasma NEFA concentrations increased $18-260 \%$ above baseline. 


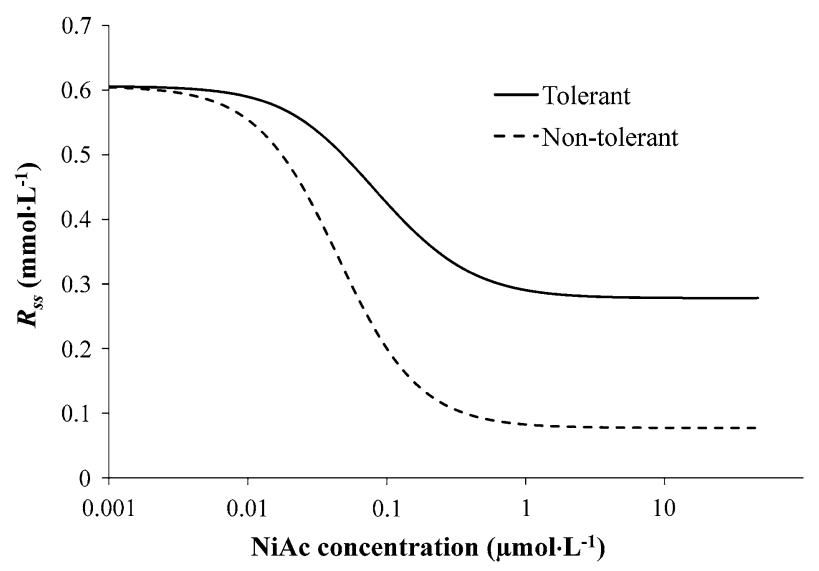

Fig. 9 Simulated steady state plasma NiAc concentration versus plasma NEFA concentration $\left(R_{s s}\right)$. The solid line represents the tolerant system according to Eq. 15, and the dashed line the non-tolerant system according to Eq. 17. The final parameter estimates from Table 2 were used to draw the two curves

During the 300 min infusions the slowly developing tolerance gave rise to increasing NEFA concentrations despite constant exposure to NiAc. Finally, there was an oscillatory pattern during the decline of the rebound, particularly after the extended infusions.

To characterize NiAc-induced changes in plasma NEFA concentrations, we have developed a mathematical model based on feedback coupled to a series of moderator transit compartments, with the first moderator compartment acting negatively on the formation of response, and the last acting positively on loss of response. It is well-known that insulin is involved in the regulation of NEFA; rapidly by inhibiting the hydrolysis of TG to NEFA and glycerol [24, 25, 27] and by stimulating the re-esterification of NEFA to TG by means of a more slowly regulated process [24-27]. The transit compartment approach is a well established tool for describing delays caused by transduction processes between a biosignal and dynamic responses [30]. The proposed feedback model mimics the observed complex NEFA pattern with regard to tolerance during NiAc exposure, rebound, and the oscillatory return of NEFA towards its predose baseline concentration upon cessation of NiAc treatment. The parameters showed low correlation and shrinkage and could be estimated with high precision. No obvious trends were seen in the goodness-of-fit-plots (Fig. 8).

The NEFA response-time data were initially analyzed by means of a pool/ precursor model [10-12]. Since the basic pool/precursor model predicts equal response and rebound areas, cannot separate response at steady state from baseline, lacks the characteristics of a dampened system, and gave systematic deviations between observed and predicted data, it was discarded at an early state. An alternative way to describe the dual-action of the moderator (e.g. insulin) mechanistically would be to include two parallel moderators, each with its own rate constants. One disadvantage of the latter approach compared to the model in Fig. 1 is the increased number of parameters. However, the model with parallel 
moderators also failed to adequately mimic the oscillations seen in the rebound after extended infusions of NiAc.

The population mean estimate of NiAc potency $\left(I C_{50}\right)$ was $45 \mathrm{nmol} \mathrm{L}{ }^{-1}( \pm 7.2 \%$ RSE) which was six times higher than the endogenous concentration estimate of $\mathrm{NiAc}\left(7 \mathrm{nmol} \mathrm{L}{ }^{-1}\right)$. The range of individually predicted endogenous NiAc concentrations was $5-15 \mathrm{nmol} \mathrm{L}^{-1}$ and below the NiAc potency. The lower limit of plasma NEFA concentrations was captured by means of a separate parameter $k_{\text {cap }}$, mimicking the non-adipocyte release of NEFA into plasma. This parameter represents the hydrolysis of TG to NEFA and glycerol in the capillaries and is affected neither by NiAc nor by the moderator. Because this lower limit is seen following administration of other GPR109A agonists (e.g. acipimox [31]), it is suggested to be a system- rather than a drug-specific parameter, so replacing $k_{c a p}$ with $I_{\text {max }}$ in the drug mechanism function (Eq. 2) would not be mechanistically correct. Simultaneous estimation of $k_{c a p}$ and $I_{\max }$ would be difficult since both of the parameters are determined from the minimum response and will therefore be highly correlated unless data of both full and partial agonists are evaluated simultaneously. Hence, the $I_{\max }$ parameter was fixed to unity in the analysis. The exponent $p$ of the first moderator $M_{1}$, which was added to amplify the impact of the negative feedback, was estimated to $1.2( \pm 6.6 \% \mathrm{RSE})$, which is statistically significant different from unity (1) based on the $95 \%$ confidence interval of the parameter estimate. The halflife of the primary effect $t_{1 / 2 \text {, kout }}$ was predicted to $1.7 \mathrm{~min}$ and the half-life of tolerance development $t_{1 / 2, k t o l}$ to $12 \mathrm{~min}$. Although there is no generic design of experiments aimed at capturing tolerance development, multiple provocations for at least $3-4 \cdot t_{1 / 2, k t o l}$, coupled to washout dynamics with sufficient baseline data, are commonly recommended [32].

Ideally, the number of transit compartments should be estimated as a parameter in the model, as this makes it possible to add interindividual variability of the number of compartments, as shown by Sun and Jusko [30] and Savic et al. [33]. However, because their approaches were based on the assumption that there was no continuous input to the first transit compartment, they could not be applied to our system. Thus, the optimal number of compartments was estimated to be eight by manually increasing $N$ until the best fit and minimum OFV was established. By increasing $N$, the oscillatory behavior of the response became more pronounced. When $N$ was less than six, the oscillatory rebound was not adequately captured, which also resulted in an increased OFV.

In the present analysis tolerance was assumed to be due to physiological adaptation or homeostatic counter-regulation, originating from the reduction in the plasma concentration of NEFA. An effective way to show the extent of tolerance is to derive the concentration-response relationship at equilibrium. That the steady state condition of the system exhibiting tolerance differed from the non-tolerant system can be seen in Fig. 9, with the equilibrium curve of the tolerant system being shallower and shifted to the right. The size of the shift provides a convenient and informative measurement of the impact of tolerance and is especially important for an accurate estimate of potency. If tolerance is ignored, dose predictions may fail, leading to under-prediction of the actual dose needed. 
This study aimed to refine the design used for routine compound screening. Our earlier experiments [18] were not designed to support a quantitative analysis of NEFA turnover, and also lacked NiAc concentration-time data. The amount of drug in the biophase was assumed to drive the pharmacodynamics and a first-order elimination rate constant of NiAc from the biophase was estimated in the modeling of pharmacodynamic data [18]. The potency estimate from that study [18] appeared to be similar to that in the present analysis $\left(I C_{50}\right.$ value of $38 \mathrm{nmol} \mathrm{L}{ }^{-1} \pm 31 \% \mathrm{RSE}$ versus $45 \mathrm{nmol} \mathrm{L}{ }^{-1} \pm 7.2 \% \mathrm{RSE}$, respectively). However, the more robust design in the present study yielded higher parameter precision and valuable information about the system, such as the slowly developing tolerance and the oscillatory decline in the rebound following long infusions of NiAc. Furthermore, determining the plasma exposure to NiAc gave direct information about potency and efficacy, and their relation to safety, and established target engagement.

Overall, the individual predictions provided a good description of the shape of the observed responses. Not only was parameter precision high, but the parameters obtained were independent of the extent and duration of exposure. Ideally, all components known to be involved in the regulation of NEFA (e.g. insulin, glucose, TG, growth hormone, and glucagon) should be observed experimentally and modeled simultaneously with the NEFA-time data. However, for the purpose of assessing the rate and extent of tolerance development and rebound after acute dosing, the present model suffices.

This study shows that it is possible to predict the time-course of drug effects in vivo in situations where feedback mechanisms are operative. The proposed model may serve as a preclinical tool for predicting and analyzing changes in plasma NEFA concentrations after treatment with NiAc or NiAc analogues [28]. Sparse sampling of a follow-up compound may suffice, provided the system parameters have been accurately characterized. This allows a smoother drug selection process.

\section{Appendix A: disposition of NiAc}

The disposition of NiAc at baseline was described by:

$$
\left\{\begin{aligned}
V_{C} \frac{d C_{p}}{d t}= & S y n t-\frac{V_{\max _{1}}}{K_{m_{1}}+C_{p_{\text {Baseline }}}} C_{p_{\text {Baseline }}}-\frac{V_{\max _{2}}}{K_{m_{2}}+C_{p_{\text {Baseline }}}} C_{p_{\text {Baseline }}} \\
& -C l_{d} C_{p_{\text {Baseline }}}+C l_{d} C_{t_{\text {Baseline }}}=0 \\
V_{t} \frac{d C_{t}}{d t}= & C l_{d} C_{p_{\text {Baseline }}}-C l_{d} C_{t_{\text {Baseline }}}=0
\end{aligned}\right.
$$

Equation 9 were rearranged to yield the expression:

$$
\text { Synt }-\frac{V_{\max _{1}}}{K_{m_{1}}+C_{p_{\text {Baseline }}}} C_{p_{\text {Baseline }}}-\frac{V_{\max _{2}}}{K_{m_{2}}+C_{p_{\text {Baseline }}}} C_{p_{\text {Baseline }}}=0
$$

or 


$$
\begin{aligned}
& \left(\text { Synt }-V_{\max _{1}}-V_{\max _{2}}\right) C_{p_{\text {Baseline }}}^{2}+\left(\operatorname{Synt}\left(K_{m_{1}}+K_{m_{2}}\right)-V_{\max _{1}} K_{m_{2}}-V_{\max _{2}} K_{m_{1}}\right) C_{p_{\text {Baseline }}} \\
& \quad+\text { SyntK }_{m_{1}} K_{m_{2}}=0
\end{aligned}
$$

The solution to this quadratic equation is:

$$
C_{p_{\text {Baseline }}}=\frac{-b \pm \sqrt{b^{2}-4 a c}}{2 a}
$$

where

$$
\begin{aligned}
& a=\text { Synt }-V_{\max _{1}}-V_{\max _{2}}, b=\operatorname{Synt}\left(K_{m_{1}}+K_{m_{2}}\right)-V_{\max _{1}} K_{m_{2}}-V_{\max _{2}} K_{m_{1}}, \\
& c=S_{\text {Synt }} K_{m_{1}} K_{m_{2}}
\end{aligned}
$$

There was only one positive root to Eq. 12, providing the estimate of $C_{\text {PBaseline }}$.

\section{Appendix B: derivation of steady state conditions}

Derivations were used to determine the steady state response $R_{s s}$ and the plasma concentration $C_{p}$ for the tolerant model (Eqs. 3, 4) and for the corresponding nontolerant model:

$$
\frac{d R}{d t}=k_{\text {in }} I\left(C_{p}\right)+k_{\text {cap }}-k_{\text {out }} R
$$

where the drug mechanism function $I\left(C_{p}\right)$ is given by Eq. 2 .

For the model involving tolerance, formulated in Eqs. 3 and 4, the steady state values for the response $R$ and the moderators $M_{i}(i=1, \ldots, N)$ are given by:

$$
R=M_{1}=\cdots=M_{N}=R_{s s}
$$

where $R_{s s}$ is the unique solution of the equation:

$$
\frac{k_{\text {in }}}{R_{s s}^{p}} I\left(C_{p}\right)+k_{\text {cap }}-k_{\text {out }} R_{s s}^{2}=0
$$

in which

$$
k_{\text {in }}=\left(k_{\text {out }} R_{0}^{2}-k_{\text {cap }}\right) R_{0}^{p}
$$

and $R_{0}$ is the baseline response.

In the absence of tolerance, $R_{s s}$ needs to satisfy:

$$
k_{\text {in }} I\left(C_{p}\right)+k_{\text {cap }}-k_{\text {out }} R_{s s}=0
$$

where

$$
k_{\text {in }}=k_{\text {out }} R_{0}-k_{\text {cap }}
$$

Note that since $I_{\max }=1$, it follows from the definition (Eq. 2) of $I\left(C_{p}\right)$ that $I\left(C_{p}\right) \rightarrow$ 0 as $C_{p} \rightarrow \infty$. This implies that: 


$$
\begin{aligned}
& \lim _{C_{p} \rightarrow \infty} R_{s s}\left(C_{p}\right)=\left(\frac{k_{\text {cap }}}{k_{\text {out }}}\right)^{1 / 2} \text { tolerant } \\
& \lim _{C_{p} \rightarrow \infty} R_{\text {ss }}\left(C_{p}\right)=\frac{k_{\text {cap }}}{k_{\text {out }}} \text { non }- \text { tolerant }
\end{aligned}
$$

\section{Appendix C: derivation of $\boldsymbol{k}_{t o l}$ and its half-life}

If exposure is sufficiently long, the response and moderators will all reach their steady state $R_{s s}=M_{s s}$, where $M_{s s}$ is the steady state for each of the $N=8$ moderators. Upon washout, because of the delay caused by the cascade, it takes time for the last moderator, $M_{8}$ to depart from its steady state, so $M_{8}(t) \approx M_{s s}$. Therefore, in the early stages of the washout period, the dynamics is effectively described by the system:

$$
\left\{\begin{aligned}
\frac{d R}{d t} & =\frac{k_{\text {in }}}{M_{1}^{p}}+k_{\text {cap }}-k_{\text {out }} R M_{s s} \\
\frac{d M_{1}}{d t} & =k_{\text {tol }}\left(R-M_{1}\right)
\end{aligned}\right.
$$

which involves $R$ and $M_{1}$ only. It is this simpler system which describes the rebound and the initial period of the return to baseline.

Derivation of the baseline

It is important to notice that the baseline $R_{b}$ of the system (i.e., immediately after washout; Eq. 21) is different, and in fact higher, than the baseline $R_{0}$ of the full system described by Eqs. 3 and 4 . To demonstrate this, we observe that $R_{b}$ and $R_{0}$ satisfy the following expressions, respectively:

$$
\begin{aligned}
\frac{k_{\text {in }}}{R_{b}^{p}}+k_{\text {cap }}-k_{\text {out }} R_{b} M_{\text {ss }} & =0 \\
\frac{k_{\text {in }}}{R_{0}^{p}}+k_{\text {cap }}-k_{\text {out }} R_{0}^{2} & =0
\end{aligned}
$$

so that:

$$
\frac{k_{\text {in }}}{R_{b}^{p}}-\frac{k_{\text {in }}}{R_{0}^{p}}=k_{\text {out }}\left(R_{b} M_{s s}-R_{0}^{2}\right)
$$

Suppose that $R_{b} \leq R_{0}$, then the left-hand side of Eq. 23 is non-negative, whilst the right-hand side is negative, because $M_{s s}<R_{0}$. Because this is a contradiction, $R_{b}>R_{0}$, as asserted.

Derivation of $k_{t o l}$

If $k_{\text {tol }}$ is small compared to $k_{\text {out }} M_{s s}$, the proposal of Gabrielsson and Peletier [17] shows that $R$ and $M_{1}$ are in quasi-equilibrium; that is, 


$$
R(t) \approx \frac{1}{k_{\text {out }} M_{s s}}\left(\frac{k_{\text {in }}}{M_{1}^{p}(t)}+k_{\text {cap }}\right) \approx \frac{\tilde{R}_{b}^{p+1}}{M_{1}^{p}(t)}
$$

where

$$
\tilde{R}_{b}^{p+1}=\frac{k_{\text {in }}}{k_{\text {out }} M_{\text {ss }}}
$$

because $k_{\text {cap }}$ is small. Note that $\tilde{R}_{b} \approx R_{b}$.

Substituting this approximate expression for $R$ into the equation for $M_{1}$ in Eq. 21 , gives:

$$
\frac{d M_{1}}{d t}=k_{t o l}\left(\frac{\tilde{R}_{b}^{p+1}}{M_{1}^{p}}-M_{1}\right)
$$

By multiplying this equation by $M_{1}^{p}$ and writing $M_{1}^{p+1}=y$, we obtain:

$$
\frac{d y}{d t}=(1+p) k_{t o l}\left(\tilde{R}_{b}^{p+1}-y\right)
$$

so that:

$$
y(t)=M_{1}^{p+1}(t)=\tilde{R}_{b}^{p+1}\left(1+B e^{-(p+1) k_{t o l} t}\right)
$$

where $\mathrm{B}$ is a constant factor. Therefore, as $t \rightarrow \infty$ :

$$
M_{1}(t)-\tilde{R}_{b}=O\left(e^{-(p+1) k_{t o l} t}\right)
$$

Thus, $M_{1}(t)$ and hence $R(t)$, converge towards the local baseline with a half-life given approximately by:

$$
t_{1 / 2, k_{t o l}} \approx \frac{\ln (2)}{(p+1) k_{t o l}}
$$

We assume that an initial estimate of $k_{t o l}$ may be approximated from the log-linear decline of NEFA post-rebound time-course.

We conclude that by estimating the half-life of the return to baseline after the rebound, we can obtain a first estimate of $k_{t o l}$.

Open Access This article is distributed under the terms of the Creative Commons Attribution Noncommercial License which permits any noncommercial use, distribution, and reproduction in any medium, provided the original author(s) and source are credited.

\section{References}

1. Gupta DK, Jewitt DE, Young R, Hartog M, Opie LH (1969) Increased plasma-free-fatty-acid concentrations and their significance in patients with acute myocardial infarction. Lancet 294(7632): 1209-1213

2. Hendrickson SC, St. Louis JD, Lowe JE, Abdelaleem S (1997) Free fatty acid metabolism during myocardial ischemia and reperfusion. Mol Cell Biochem 166(1-2):85-94 
3. Carlson LA, Orö L (1962) The effect of nicotinic acid on the plasma free fatty acid: demonstration of a metabolic type of sympathicolysis. Acta Med Scand 172:641-645

4. Carlson LA (1963) Studies on the effect of nicotinic acid on catecholamine stimulated lipolysis in adipose tissue in vitro. Acta Med Scand 173:719-722

5. Canner PL, Berge KG, Wenger NK (1986) Fifteen year mortality in Coronary Drug Project patients: long-term benefit with niacin. J Am Coll Cardiol 8(6):1245-1255

6. Carlson LA, Rosenhamer G (1988) Reduction of mortality in the Stockholm ischaemic heart disease secondary prevention study by combined treatment with clofibrate and nicotinic acid. Acta Med Scand 223(5):405-418

7. Carlson LA (2005) Nicotinic acid: the broad-spectrum lipid drug. A 50th anniversary review. J Intern Med 258(2):94-114

8. Offermanns S (2006) The nicotinic acid receptor GPR109A (HM74A or PUMA-G) as a new therapeutic target. Trends Pharmacol Sci 27(7):384-390

9. Pereira JN (1967) The plasma free fatty acid rebound induced by nicotinic acid. J Lipid Res 8(3):239-244

10. Licko V, Ekblad EBM (1992) Dynamics of a metabolic system: what single-action agents reveal about acid secretion. Am J Physiol 262(3):581-592

11. Bauer JA, Fung HL (1994) Pharmacodynamic models of nitroglycerin-induced hemodynamic tolerance in experimental heart failure. Pharm Res 11(6):816-823

12. Sharma A, Ebling WF, Jusko WJ (1998) Precursor-dependent indirect pharmacodynamic response model for tolerance and rebound phenomena. J Pharm Sci 87(12):1577-1584

13. Zannikos PN, Rohatagi S, Jensen BK (2001) Pharmacokinetic-pharmacodynamic modeling of the antilipolytic effects of an adenosine receptor agonist in healthy volunteers. J Clin Pharmacol 41(1):61-69

14. Wakelkamp M, Alván G, Gabrielsson J, Paintaud G (1996) Pharmacodynamic modeling of furosemide tolerance after multiple intravenous administration. Clin Pharmacol Ther 60(1):75-88

15. Zuideveld KP, Maas HJ, Treijtel N, Hulshof J, van der Graaf PH, Peletier LA, Danhof M (2001) A set-point model with oscillatory behavior predicts the time course of 8-OH-DPAT-induced hypothermia. Am J Physiol Regul Integr Comp Physiol 281(6):2059-2071

16. Bundgaard C, Larsen F, Jorgensen M, Gabrielsson J (2006) Mechanistic model of acute autoinhibitory feedback action after administration of SSRIs in rats: application to escitalopram-induced effects on brain serotonin levels. Eur J Pharm Sci 29(5):209-219

17. Gabrielsson J, Peletier LA (2007) A nonlinear feedback model capturing different patterns of tolerance and rebound. Eur J Pharm Sci 32(2):85-104

18. Isaksson C, Wallenius K, Peletier LA, Toresson H, Gabrielsson J (2009) Turnover modeling of nonesterified fatty acids in rats after multiple intravenous infusions of nicotinic acid. Dose-Response 7(3):247-269

19. Popovic V, Popovic P (1960) Permanent cannulation of aorta and vena cava in rats and ground squirrels. J Appl Physiol 15(4):727-728

20. McCreanor G, Bender DA (1986) The metabolism of high intakes of tryptophan, nicotinamide and nicotinic acid in the rat. Br J Nutr 56(3):577-586

21. Soga T, Kamohara M, Takasaki J, Matsumoto SI, Saito T, Ohishi T, Hiyama H, Matsuo A, Matsushime H, Furuichi K (2003) Molecular identification of nicotinic acid receptor. Biochem Biophys Res Commun 303(1):364-369

22. Tunaru S, Kero J, Schaub A, Wufka C, Blaukat A, Pfeffer K, Offermanns S (2003) PUMA-G and HM74 are receptors for nicotinic acid and mediate its anti-lipolytic effect. Nat Med 9(3):352-355

23. Wise A, Foord SM, Fraser NJ, Barnes AA, Elshourbagy N, Eilert M, Ignar DM, Murdock PR, Steplewski K, Green A, Brown AJ, Dowell SJ, Szekeres PG, Hassalli DG, Marshall FH, Wilson S, Pike NB (2003) Molecular identification of high and low affinity receptors for nicotinic acid. J Biol Chem 278(11):9869-9874

24. Frayn KN (2003) Metabolic regulation: a human perspective. Organs and tissues. Blackwell Science Ltd, Oxford, UK

25. Frayn KN, Shadid S, Hamlani R, Humphreys SM, Clark ML, Fielding BA, Boland O, Coppack SW (1994) Regulation of fatty acid movement in human adipose tissue in the postabsorptive-to-postprandial transition. Am J Physiol Endocrinol Metab 266(3):308-317

26. Sadur CN, Eckel RH (1982) Insulin stimulation of adipose tissue lipoprotein lipase. Use of the euglycemic clamp technique. J Clin Invest 69(5):1119-1125 
27. Stralfors P, Bjorgell P, Belfrage P (1984) Hormonal regulation of hormone-sensitive lipase in intact adipocytes: identification of phosphorylated sites and effects on the phosphorylation by lipolytic hormones and insulin. Proc Natl Acad Sci USA 81(11):3317-3321

28. Levy G (1993) Integration of pharmacokinetics, pharmacodynamics and toxicokinetics in rational drug development, the case for preclinical pharmacodynamics. Plenum Press, New York

29. Iwaki M, Ogiso T, Hayashi H, Tanino T, Benet LZ (1996) Acute dose-dependent disposition studies of nicotinic acid in rats. Drug Metab Dispos 24(7):773-779

30. Sun YN, Jusko WJ (1998) Transit compartments versus gamma distribution function to model signal transduction processes in pharmacodynamics. J Pharm Sci 87(6):732-737

31. Carballo-Jane E, Gerckens LS, Luell S, Parlapiano AS, Wolff M, Colletti SL, Tata JR, Taggart AK, Waters MG, Richman JG, McCann ME, Forrest MJ (2007) Comparison of rat and dog models of vasodilatation and lipolysis for the calculation of a therapeutic index for GPR109A agonists. J Pharmacol Toxicol Methods 56(3):308-316

32. Gabrielsson J, Peletier LA (2008) A flexible nonlinear feedback system that captures diverse patterns of adaptation and rebound. AAPS J 10(1):70-83

33. Savic RM, Jonker DM, Kerbusch T, Karlsson MO (2007) Implementation of a transit compartment model for describing drug absorption in pharmacokinetic studies. J Pharmacokinet Pharmacodyn 34(5):711-726 